\title{
INFLUENCE OF MECHANICAL STIRRING ON THE CRUCIBLE DISSOLUTION RATE AND IMPURITIES DISTRIBUTION IN DIRECTIONAL SOLIDIFICATION OF MULTICRYSTALLINE SILICON
}

\author{
Alexandra Popescu, Daniel Vizman \\ Faculty of Physics, West University of Timisoara, Bd. V. Parvan 4, 300223Timisoara, Romania
}

\begin{abstract}
Article Info
Received: 08.09.2015

Accepted: 21.11.2015

Keywords: Numerical simulations, Fluid flow, Ingot casting method, Stirring, Multicrystalline silicon;
\end{abstract}

\begin{abstract}
In this study, time dependent three-dimensional numerical simulations were carried out using the STHAMAS3D software in order to understand the effects of forced convection induced by mechanical stirring of the melt, on the crucible dissolution rate and on the impurities distribution in multicrystalline silicon (mc-Si) melt for different values of the diffusion coefficient.

Numerical simulations were performed on a pilot scale furnace with crucible dimensions of $38 \times 38 \times 40 \mathrm{~cm}^{3}$. The computational domain used for the local 3D-simulations consists of melt and crystal.

The dissolution rate was estimated from the total mass of impurities that was found in the silicon melt after a certain period of time. The obtained results show that enhanced convection produced by a mechanical stirrer leads to a significant increase of the dissolution rate and also to a uniform distribution of impurities in the melt.
\end{abstract}

\section{Introduction}

One of the basic principles for maximizing the solar cell yield is to improve the collection of minority carriers, the probability for collecting them being higher if the minority carrier's lifetime is higher. The minority carrier lifetime can be affected by the impurities concentration in the wafers which can act as recombination sites.

Therefore, the multicrystalline silicon (mc-Si) properties, like diffusion length or minority carriers lifetime (properties important also for the performance of a solar cell) are correlated with the appearance and content of impurities (mainly carbon, nitrogen and oxygen), but also with the presence of extended defects (dislocations, grain boundaries) in the crystal $[1,2,3]$. The presence of carbon and nitrogen in the silicon melt during the directional solidification process, can lead to the formation of $\mathrm{SiC}$ and $\mathrm{Si}_{3} \mathrm{~N}_{4}$ precipitates $[4,5,6,7,8,9]$ which can determine the appearance of dislocations and cracks during the wafer sawing of the ingot. 
In order to improve the impurity segregation and to obtain a uniform distribution of impurities in the melt, different techniques were proposed to control the melt flow in a directional solidification configuration: use of travelling magnetic fields [10, 11], use of a combination of vertical magnetic field and electrical current [12] or a mechanical stirring [13]. Besides the beneficial influence on the impurity distribution, one should also take into account the influence of the forced convection on the solid-liquid interface and on the crucible dissolution. A previous study [6] showed that the natural convection increases the crucible dissolution rate and this effect is stronger for higher values of the diffusion coefficient. It is expected that the forced convection of mc-Si melt will lead to a stronger increase of the dissolution rate, and to a higher degree of impurification of the melt.

As it was shown previously [13], mechanical stirring can be a good option for improving the melt flow in large crucibles even for smaller values of the stirring's rotation rate. The influences of the mechanical stirring were investigated on both melt convection and interface shape, but there is no analysis on impurities distribution and crucible dissolution rates. Because these influences are of crucial importance for the applications, it is the aim of this paper to continue the investigation on mechanical stirring from [13] with focus on impurities distribution and crucible dissolution rates.

Our research is focused on the numerical study of the way the mechanical stirring of mc-Si melt influences the crucible dissolution rate and impurities distribution using different rotation rates and several diffusion coefficients. Because these coefficients are very difficult to be measured at melting temperature the numerical simulations can be affected by the errors in diffusion constants. For this study the diffusion coefficients are chosen in a range characteristic for the most present impurities in the silicon crystal: $\mathrm{C}, \mathrm{N}, \mathrm{O}$, which have the diffusion coefficients between $2-5 \cdot 10^{-8} \mathrm{~m}^{2} / \mathrm{s}[5,6,14]$.

\section{Numerical model}

A simplified local three-dimensional model has been developed for the directional solidification process of mc-Si. The numerical simulations have been made on a pilot scale furnace with dimensions of $38 \times 38 \times 40 \mathrm{~cm}^{3}$ corresponding to the $\mathrm{G} 2$ (second generation) standard crucible. The computational domain consists of silicon melt and crystal, as shown in Figure 1. The mechanical stirrer is immersed $15 \mathrm{~cm}$ in the melt, and is rotated at different frequencies. 


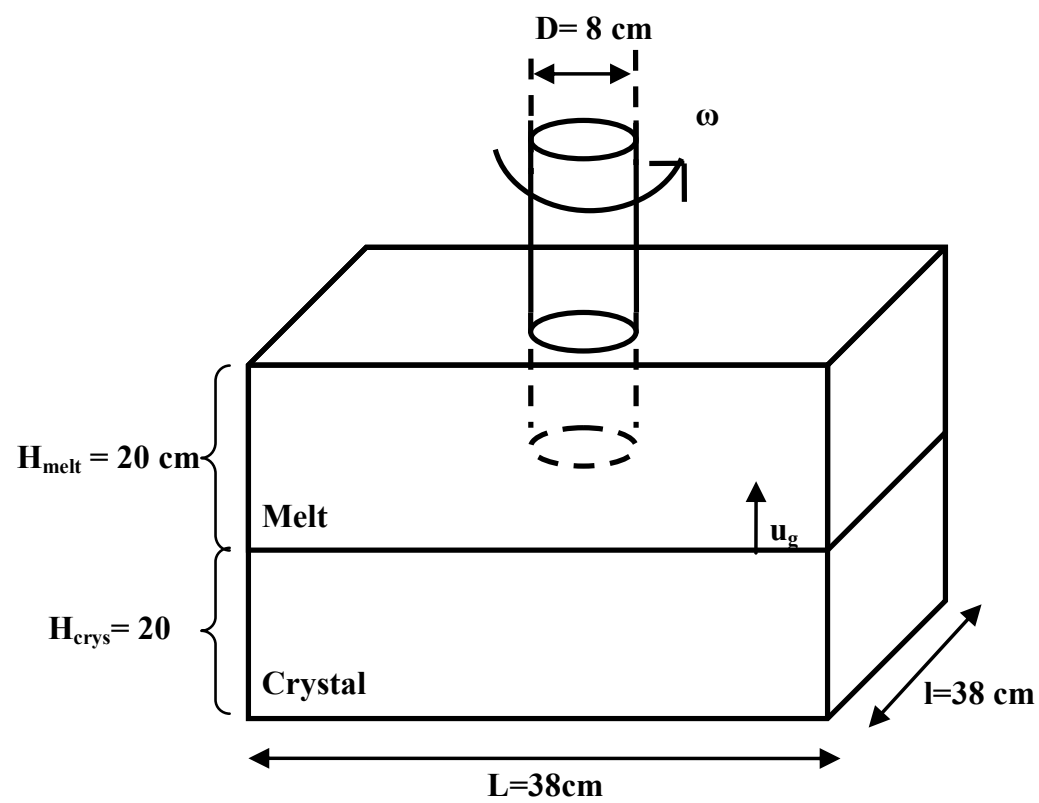

Fig. 1. Schematic view of the crucible and stirrer

The melt flow is described by the three-dimensional equations for mass, momentum, heat transport and diffusion. The Boussinesq approximation for an incompressible Newtonian fluid is taken into consideration:

$$
\begin{gathered}
\frac{\partial}{\partial y_{i}}\left(\rho u_{i}\right)=0 \\
\rho \frac{\partial u_{i}}{\partial t}=-\frac{\partial}{\partial y_{j}}\left[\rho u_{i} u_{j}-\mu\left(\frac{\partial u_{i}}{\partial y_{j}}+\frac{\partial u_{j}}{\partial y_{i}}\right)\right]-\frac{\partial p}{\partial y_{i}}+f_{i} \\
\frac{\partial}{\partial t}\left(\rho C_{p} T\right)=-\frac{\partial}{\partial y_{j}}\left(\rho C_{p} u_{j} T-k \frac{\partial T}{\partial y_{j}}\right) \\
\frac{\partial c}{\partial t}=-\frac{\partial}{\partial y_{j}}\left(u_{j} c-D \frac{\partial c}{\partial y_{j}}\right)
\end{gathered}
$$

where $\rho$ is the fluid density, $u_{i}$ is the flow velocity, $p$ the pressure, $C_{p}$ the heat capacity, $k$ the thermal conductivity, $c$ is the impurities concentration and $D$ is the diffusion coefficient. $f_{i}$ is the source term for the momentum equation and can be written as:

$$
f_{i}=f_{g, i}+f_{r o t, i}
$$

where: 
$f_{g, i}=-\rho T_{r e f} g_{i} \beta\left(T-T_{r e f}\right)$ is the Boussinesq approximation for an incompressible fluid and $f_{\text {rot }, i}$ corresponds to the forced convection produced by the mechanical stirrer.

A detailed description of the model was presented in [13] and it mainly consists of the following assumptions:

i) The mechanical stirrer is cylindrically shaped and is $8 \mathrm{~cm}$ in diameter (D). It is considered to be centered on the vertical symmetry axis and it can be rotated with different angular velocities during the solidification process.

ii) The stirring device is not considered as being physically present in the melt, but its effect was considered by imposing the presence of an azimuthally volumetric force $\left(f_{r o t, i}\right)$ in each point of the region where the stirrer is located.

The force has been chosen as:

$$
f_{r o t, i}=\left(\rho \omega \cdot \omega_{i}\right) r_{i}
$$

where $\omega$ is the stirrer's angular velocity and $r_{i}$ is the distance from the $i$-th point to the stirrer axis.

The melt velocity on the corresponding boundaries at the crucible and at the crystal are set to $u_{i}=0$. Along the surface of the melt a "no shear stresses" condition has been imposed. On the lateral walls and on the bottom the temperature has been fixed corresponding to a temperature gradient of $3 \mathrm{~K} / \mathrm{cm}$ in the melt and $10 \mathrm{~K} / \mathrm{cm}$ in the crystal, typical values used in a silicon directional solidification process. The growth rate is set to $u_{g}=1 \mathrm{~cm} / \mathrm{h}$ for all the computations. At the melt free surface a radiative heat exchange to the ambient temperature of $T_{r e f}=1785 \mathrm{~K}$ has been applied:

$$
-k_{L} \frac{\partial T}{\partial n}=\varepsilon \sigma\left(T^{4}-T_{r e f}^{4}\right)
$$

where $\varepsilon$ is the thermal emissivity of the silicon melt and $\sigma$ is the Stefan-Boltzmann constant.

At the solid-liquid interface the latent heat production is considered:

$$
k_{S} \frac{\partial T}{\partial n}-k_{L} \frac{\partial T}{\partial n}=\rho_{S} u_{g} \Delta H
$$

where $k_{S}$ and $k_{L}$ are the thermal conductivities of solid and liquid silicon, and $\Delta H$ is the latent heat.

In the crystal no concentration has been computed. On the lateral walls the concentration has been set to $9 \cdot 10^{24}$ atoms $/ \mathrm{m}^{3}$ which represents the solubility limit of carbon in silicon [6]. A no-flux condition has been imposed for the impurities at the melt surface and 
at the solid-liquid interface. The initial impurities concentration has been set to zero in the whole melt volume. This assumption is valid for a pure silicon melt. The impurities injected in the melt by the stirrer are neglected (the area of the stirrer is approx. 10\% from the total area of the crucible walls). All the other material properties are given in Table 1.

Table 1. Material properties used in numerical simulations

\begin{tabular}{|ccc|}
\hline Physical property & Value & Unit \\
\hline Si melt & & \\
Density & $2.52 \cdot 10^{3}$ & $\mathrm{~kg} / \mathrm{m}^{3}$ \\
Dynamic viscosity & $7.56 \cdot 10^{-4}$ & $\mathrm{Ns} / \mathrm{m}^{2}$ \\
Thermal conductivity & 60.6 & $\mathrm{~W} / \mathrm{mK}$ \\
Specific heat & 911 & $\mathrm{~J} / \mathrm{kgK}$ \\
Coefficient of thermal expansion & $1.44 \cdot 10^{-4}$ & $\mathrm{~K}^{-1}$ \\
Marangoni coefficient & $-4 \cdot 10^{-4}$ & $\mathrm{~N} / \mathrm{Km}$ \\
Si solid & & \\
Density & $2.52 \cdot 10^{3}$ & $\mathrm{~kg} / \mathrm{m}^{3}$ \\
Thermal conductivity & 22 & $\mathrm{~W} / \mathrm{mK}$ \\
Specific heat & 927 & $\mathrm{~J} / \mathrm{kgK}$ \\
Coefficient of thermal expansion & $1.44 \cdot 10^{-4}$ & $\mathrm{~K}-1$ \\
Latent heat & $158.7 \cdot 10^{4}$ & $\mathrm{~J} / \mathrm{kg}$ \\
\hline
\end{tabular}

Time dependent computations have been performed using the STHAMAS3D software developed at the Crystal Growth Laboratory in Erlangen and has already been used to simulate Czochralski configurations [14] and directional solidification processes [15].

The block-structured grid is subdivided into two blocks, one for the melt and one for the crystal, with a local grid refinement on the walls to solve the boundary layers. The grid consists of 396000 control volumes which are enough to describe the main characteristics of the flow. In [15] the dependence of z-component of the flow velocity along the central vertical line for different grid size was analyzed. It was shown that at this grid refinement level there is almost no dependence of the solution on the grid refinement.

In order to get realistic solutions starting from an arbitrary solution, the simulation extended over $3600 \mathrm{~s}$ in real time with a time step of $0.2 \mathrm{~s}$.

\section{Results and Discussions}


In order to understand the influence of forced convection generated by a mechanical stirrer on the crucible dissolution rate and on the transport of the impurities originating from the crucible, two stirrer's rotational velocity $5 \mathrm{rpm}$ and $10 \mathrm{rpm}$ and the following values for diffusion coefficient: $\left\{0.5 \cdot 10^{-8} \mathrm{~m}^{2} / \mathrm{s} ; 2 \cdot 10^{-8} \mathrm{~m}^{2} / \mathrm{s} ; 3 \cdot 10^{-8} \mathrm{~m}^{2} / \mathrm{s} ; 5 \cdot 10^{-8} \mathrm{~m}^{2} / \mathrm{s}\right\}$ were considered. The simulations extended over one hour in real time, with the mass of impurities being computed every $200 \mathrm{~s}$.

In a previous study [13], the influence of the same type of stirrer on the solid-liquid interface and on the melt convection has been investigated for different values of the stirrer's rotation rate. It was found that, as the rotation frequency increases, the melt mixing is improved both horizontally and vertically. In the present study a careful analysis of the mixing process is carried out.

Convection in a squared crucible was careful investigated in [16] and it was clearly shown that, in a vertical section, in the middle of the mould there are two main convection zones: one near the solid-liquid interface (generated by the interface bending) and one near the melt free surface (generated by the radiative heat transfer) with a more calm zone between them. Therefore it is expected the impurities distribution to be influenced by this convection patterns.

Figure 2(a) (for $\mathrm{D}=0.5 \cdot 10^{-8} \mathrm{~m}^{2} / \mathrm{s}$ ) and Figure 4(a) (for $\mathrm{D}=5 \cdot 10^{-8} \mathrm{~m}^{2} / \mathrm{s}$ ) clearly show that the impurities are trapped in two zones: one near the solid-liquid interface and one near the free surface, with a higher concentration near the solid-liquid interface. Between the two zones there is a zone with a lower level of impurities. When a rotational velocity of $5 \mathrm{rpm}$ is present, the concentration is more homogeneous in the whole vertical section (see Figure 2(b) and 4(b)). The homogeneity is increasing with the increase of the rotational velocity to 10 rpm (Figure 2(c) and 4(c)). Also the diffusion coefficient seems to influence the homogeneity in the vertical section: for the same rotation rate the concentration distribution is more homogeneous for a low diffusion coefficient $\left(D=0.5 \cdot 10^{-8} \mathrm{~m}^{2} / \mathrm{s}\right)$ Figure 2(b) in comparison with a higher diffusion coefficient $\left(D=5 \cdot 10^{-8} \mathrm{~m}^{2} / \mathrm{s}\right)$ as shown in Figure $4(\mathrm{~b})$. Because the level of impurities in the melt is strongly dependent on the diffusion coefficient, we used different scale for concentration for different diffusion coefficients. 


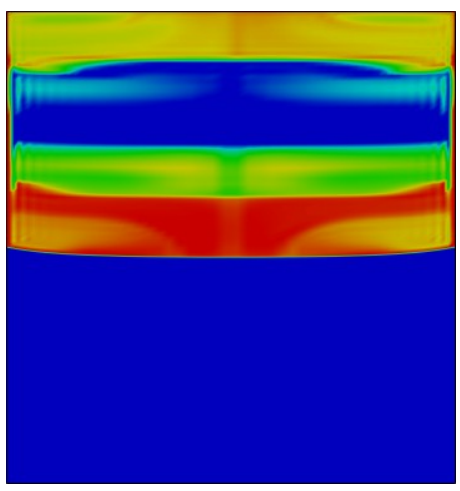

(a)

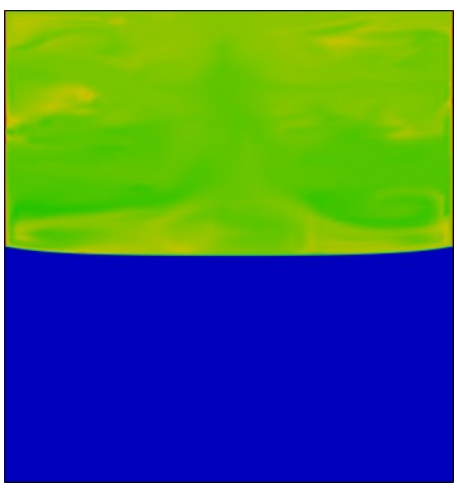

(b)

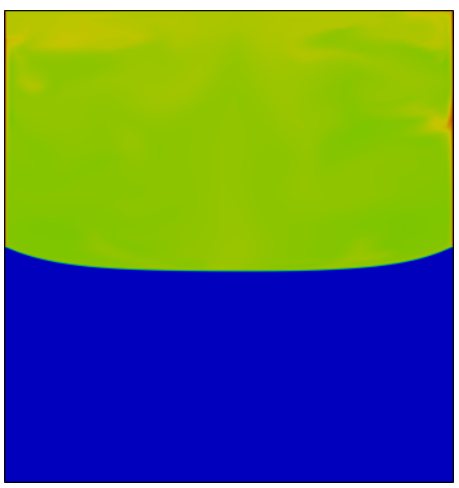

(c)

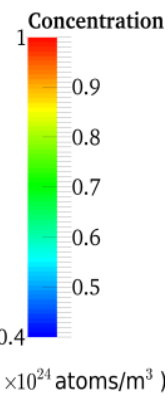

Fig. 2. Concentration distribution in a vertical section (xOz) for $\mathrm{D}=0.5 \cdot 10^{-8} \mathrm{~m}^{2} / \mathrm{s}$ and for a rotational velocity of (a) $0 \mathrm{rpm}$, (b) $5 \mathrm{rpm}$, (c) $10 \mathrm{rpm}$

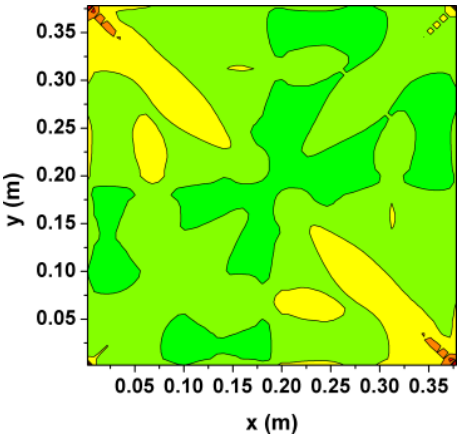

(a)

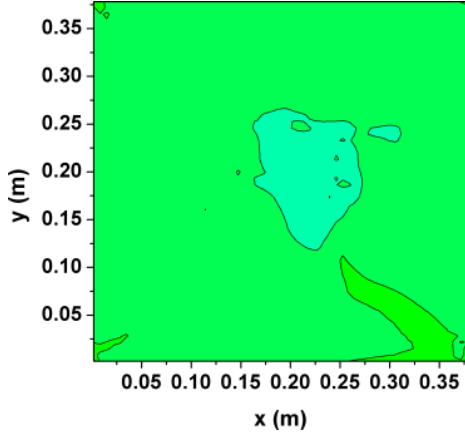

(b)

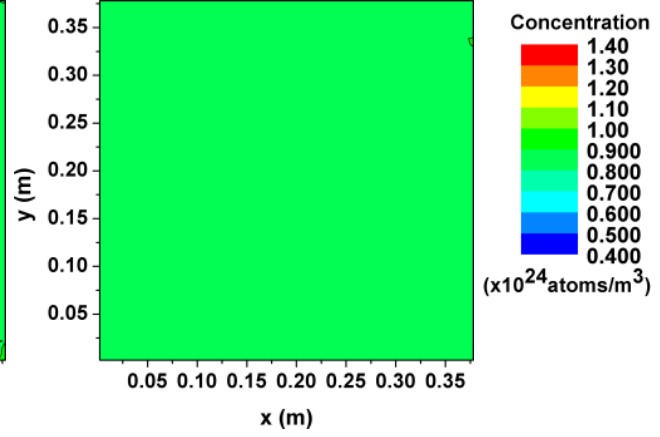

(c)

Fig. 3. Concentration distribution at the solid-liquid interface for $\mathrm{D}=0.5 \cdot 10^{-8} \mathrm{~m}^{2} / \mathrm{s}$ and for a rotational velocity of (a) $0 \mathrm{rpm}$, (b) $5 \mathrm{rpm}$, (c) $10 \mathrm{rpm}$

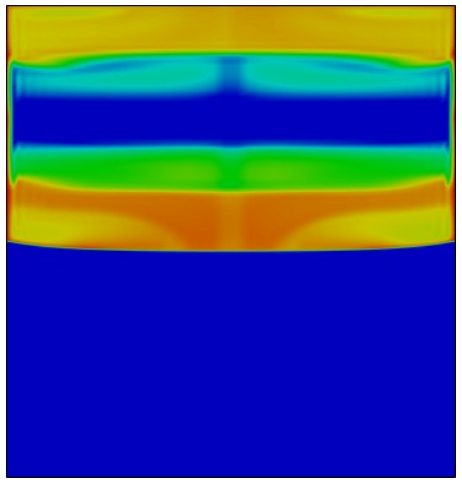

(a)

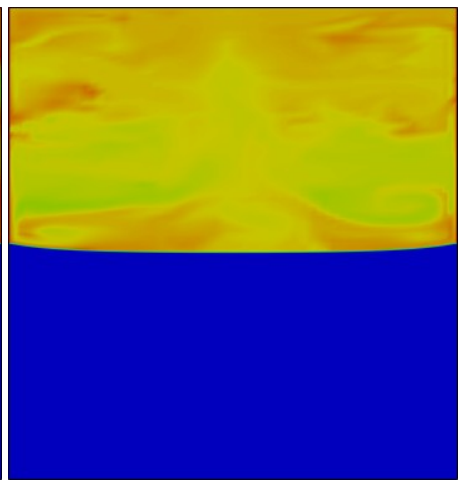

(b)

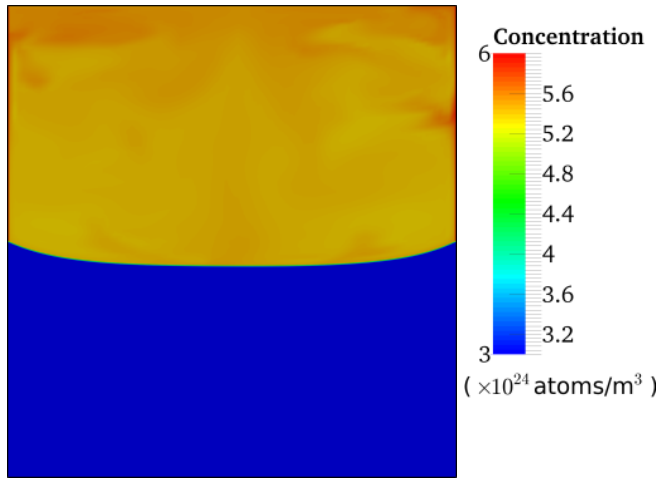

(c)

Fig. 4. Concentration distribution in a vertical section for $\mathrm{D}=5 \cdot 10^{-8} \mathrm{~m}^{2} / \mathrm{s}$ and for a rotational velocity of (a) 0 $\mathrm{rpm}$, (b) $5 \mathrm{rpm},(\mathrm{c}) 10 \mathrm{rpm}$ 


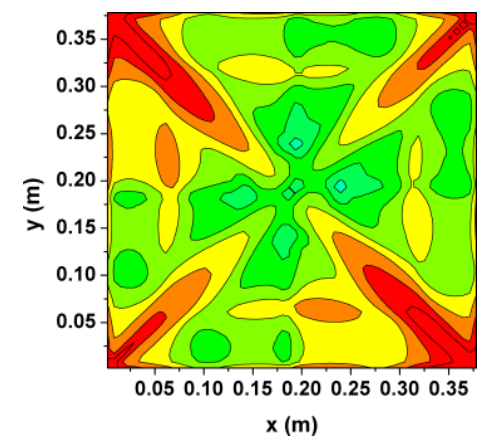

(a)

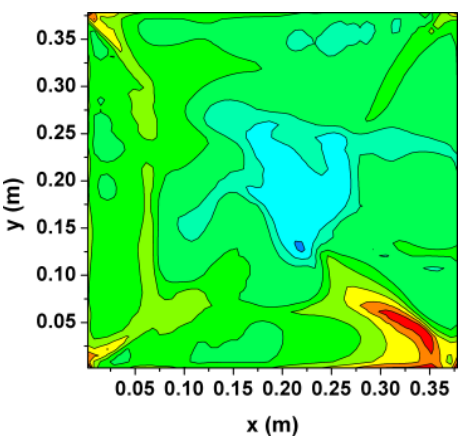

(b)

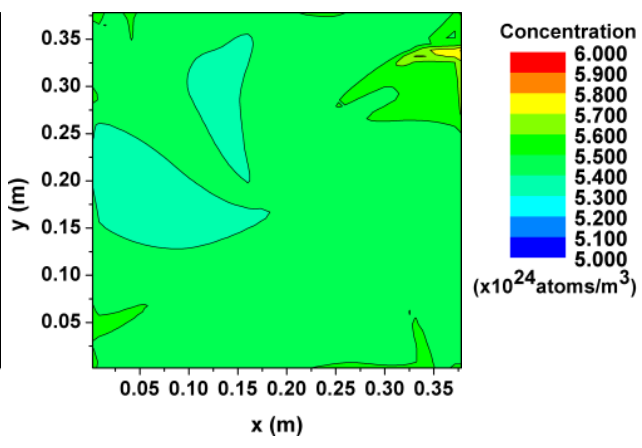

(c)

Fig. 5. Concentration distribution near the solid-liquid interface for $D=5 \cdot 10^{-8} \mathrm{~m}^{2} / \mathrm{s}$ for a rotational velocity of (a) $0 \mathrm{rpm}$, (b) $5 \mathrm{rpm}$, (c) $10 \mathrm{rpm}$

We choose to represent the concentration distributions (Figs. 2, 3, 4, 5) just for the extreme values of the diffusion coefficient, but the conclusions are valid also for the intermediate values of the diffusion coefficients.

Figure 6 shows that the total mass of impurities in the melt, for the same diffusion coefficient and at every time step is higher if the rotation is present.

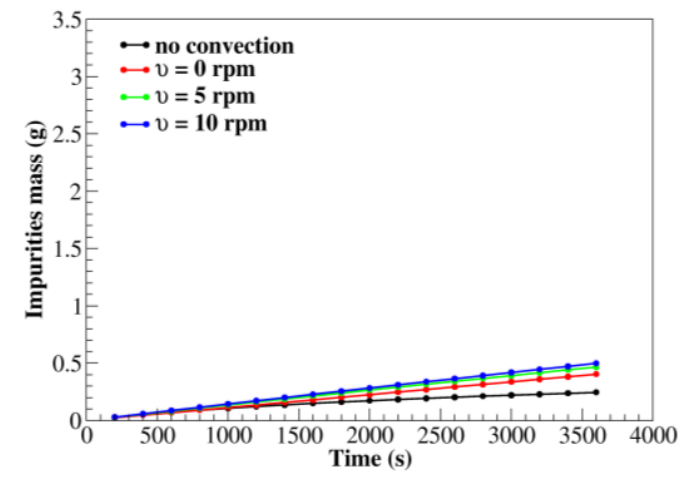

(a)

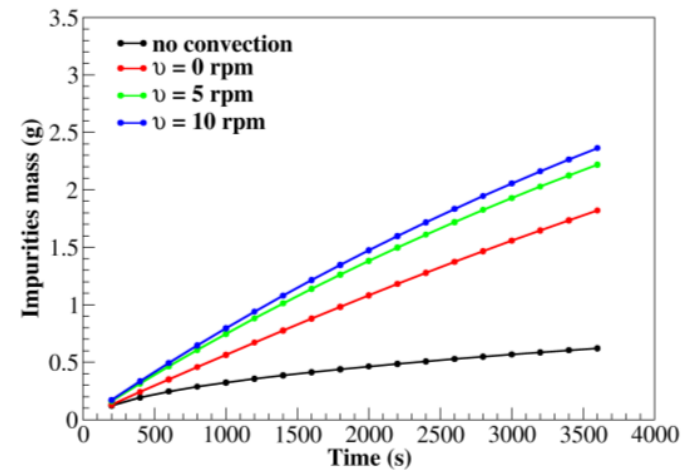

(c)

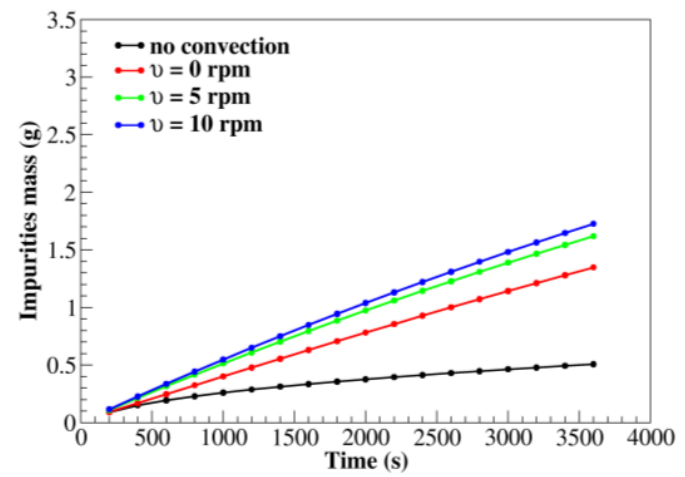

(b)

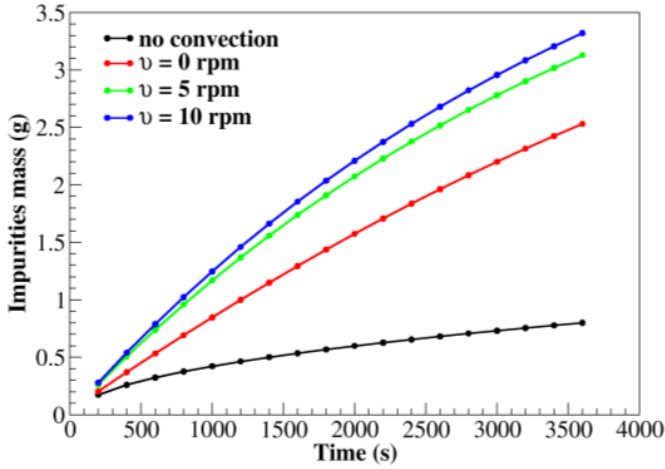

(d)

Fig. 6. Time evolution of the mass of impurities in the presence of purely diffusive regime and of the convective regime for different diffusion coefficient values: (a) $\mathrm{D}=0.5 \cdot 10^{-8} \mathrm{~m}^{2} / \mathrm{s}$; (b) $\mathrm{D}=2 \cdot 10^{-8} \mathrm{~m}^{2} / \mathrm{s}$; (c) $\mathrm{D}=3 \cdot 10^{-8} \mathrm{~m}^{2} / \mathrm{s}$; (d) $\mathrm{D}=5 \cdot 10^{-8} \mathrm{~m}^{2} / \mathrm{s}$

Nevertheless it is expected that a higher mass of impurities will be incorporated in the crystal in the case with just buoyant convection (no rotation) because the impurities are not 
homogeneous distributed in the melt and are more concentrated near the solid-liquid interface (Figure 2(a) and 4(a)). In order to see the influence of the convection (natural and forced) on the impurity mass, in Figure 6 was plotted also the pure diffusive case (black lines). It was supposed that initially the concentration of impurities in the melt was zero. It can be observed that the convection has a strong influence on the total impurity mass in the melt. The total mass increases with the increase of rotation rate and with the increase of the diffusion coefficient (Figure 6 (a)-(d)).

In a horizontal section it was shown in [16] that the symmetry of the squared crucible is reflected in the regularity of the melt flow patterns. This behavior is also obvious for the concentration distribution in the case of purely buoyant convection (see Figs. 3a, 5a): the concentration distribution is symmetric with respect to the both diagonals of the squared section (xOy). Figures 3 and 5 show the concentration distribution at the solid-liquid interface for two diffusion coefficients: $\mathrm{D}=0.5 \cdot 10^{-8} \mathrm{~m}^{2} / \mathrm{s}$ (Figure 3 ) and $\mathrm{D}=5 \cdot 10^{-8} \mathrm{~m}^{2} / \mathrm{s}$ (Figure 5) and for two rotational velocities: $5 \mathrm{rpm}$ (Figs. 3(b) and 5(b)) and $10 \mathrm{rpm}$ (Figs. 3(c) and 5(c)). Once with the melt rotation, the regularity imposed by the crucible symmetry to the concentration distribution is broken and the impurities distribution is more homogeneous. The homogeneity is increasing with the increase of the rotation rate (see Figure $3(\mathrm{~b})$ in comparison with Figure 3(c) and Figure 5(b) in comparison with Figure 5(c)) and is decreasing with the increase of diffusion coefficient (see Figure 3(b) in comparison with Figure 5(b) and Figure 3(c) in comparison with Figure 5(c)). It is also clear, looking at Figures 3 and 5 that the impurity concentration at the solid liquid interface is higher in the case of pure buoyant convection than in the case of forced convection. As we already mentioned this effect is explained by analyzing the melt convection and impurity distribution in the vertical section.

The crucible dissolution rate (DR) has been estimated from the total mass of impurities that was found in the melt after a period of time:

$$
D R=\frac{\int_{V} c d V}{t}
$$

Figure 7 shows that the crucible dissolution rate increases with the increase of the diffusion coefficient values. As a reference the purely diffusive regime (black line) was considered. It can be clearly noted that for a fixed diffusion coefficient, the crucible dissolution rate strongly increases if the convection (natural or forced) is present in comparison with the pure diffusive regime. The DR in case of forced convection is higher in comparison with the case of pure natural convection. This latter behavior is all the more 
significant as the diffusion coefficient is higher. The increase of the rotational velocity from 5 to $10 \mathrm{rpm}$ leads to a slightly increase of the dissolution rate, behavior which is more significant if the diffusion coefficient is higher.

Also, there is an increase in the dissolution rate with the increase of the diffusion coefficient, in the case of purely diffusive regime, but the increase is smaller than in the case where convection is present.

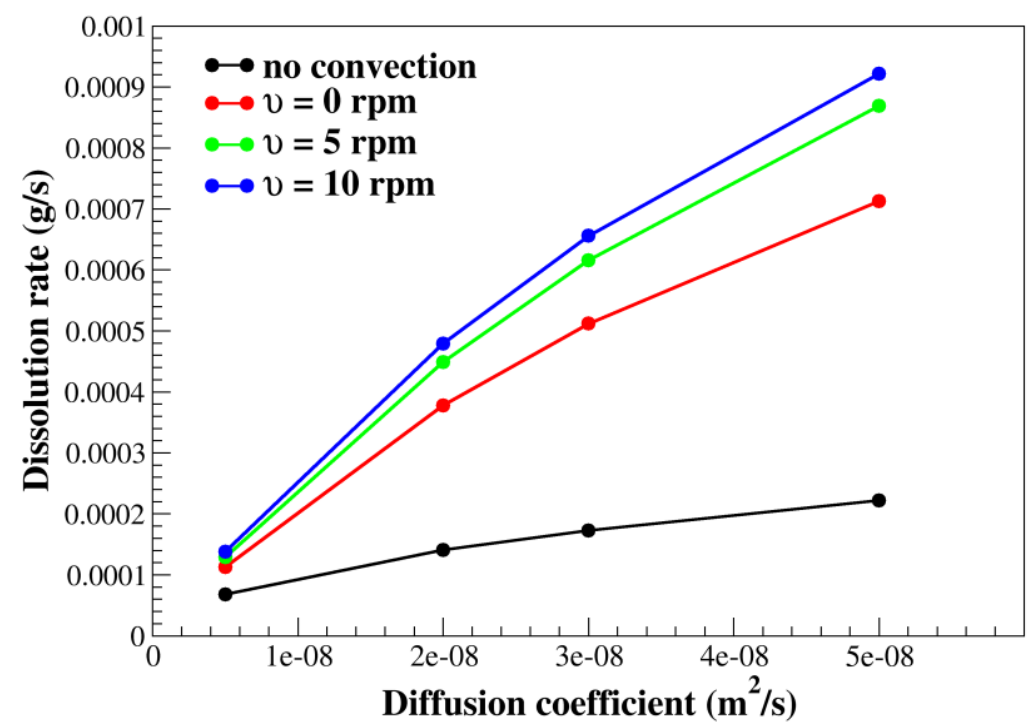

Fig. 7. Crucible dissolution rate for different diffusion coefficient values and for the purely diffusive and the convective regimes

\section{Conclusions}

The impurities distributions in a silicon melt and crucible dissolution rate were analyzed using numerical modeling in the case of forced convection generated by an idealized mechanical stirrer. The obtained results show that the mechanical stirring can lead to a significant homogenization of the impurities distribution in the melt. The crucible dissolution rate increases with the increase of the stirrer's rotation rate and is higher in the case of the forced convection than in the case of pure natural convection. Therefore also the total impurities mass in the melt is increasing. Nevertheless because the impurities are more uniform distributed in the melt, for this growth stage the impurities level at the solid liquid interface is significantly lower in the case of forced convection than in the case of natural convection.

The impurities transport is strongly dependent on the diffusion coefficient. The impurities with a higher diffusion coefficient will have a higher dissolution rate and the total homogenization is expected to take place at higher rotational rates.

In conclusion, the mechanical stirring has proved to be a good instrument for obtaining more uniformly distributed impurities in the silicon melt even for small values of the rotation 
rate $(5 \mathrm{rpm}$ and $10 \mathrm{rpm})$. Once the fundamental aspects of the mass transport under the influence of forced convection generated by a mechanical stirrer is understood, the feasibility of using mechanical stirring in the directional solidification process of silicon can be more easily analyzed. The results on the dissolution rates can be of interest for the crucible coating design which is one of the main sources of impurities in the directional solidification process of multicrystalline silicon.

\section{Acknowledgements}

This work was supported by a grant of the Romanian National Authority for Scientific Research, CNCS-UEFISCDI, project number PN-II-ID-PCE-2011-3-0789.

\section{References}

[1] T. Bounassisi et al., Prog. Photovolt. Res. Appl. 14 (2006) 513.

[2] J. Chen, T. Sekiguchi, D. Yang, F. Yin and K. Kido, J. Appl. Phys. 96 (10) (2004) 5490 .

[3] C. Reimann, M. Trempa, J. Friedrich and G. Müller, J. Cryst. Growth 312 (2010) 1510.

[4] B. Gao, S. Nakano and K. Kakimoto, J. Electrochem. Soc., 157 (2010) H153

[5] A. Lawerenz et. al., Solid State Phenom. 95-96, (2004) 501.

[6] A. Popescu and D. Vizman, Int. J. Heat Mass Transf. 54, (2011) 5540.

[7] C. Reimann, M. Trempa, T. Jung, J. Friedrich and G. Müller, J. Cryst. Growth 312 (2010) 878 .

[8] C. Reinmann, T. Jung, J. Friedrich and G. Müller, Proceedings of the 33rd IEEE PVSC, (2008) 1795.

[9] A.K. Soiland, et. al., Mater. Sci. Semicond. Process 7, 39 (2004).

[10] N. Dropka, Ch. Frank-Rotsch, W. Miller and P. Rudolph, J. Cryst. Growth 338 (2012) 208.

[11] K. Dadzis, J. Ehrig, K. Niemietz, O. Pätzold, U. Wunderwald and J. Friedrich, J. Cryst. Growth 333 (2011) 7.

[12] C. Tanasie and D. Vizman, J. Cryst. Growth 372 (2013) 1.

[13] S. Dumitrica, D. Vizman, J.-P. Garandet and A. Popescu, J. Cryst. Growth 360, 76 (2012) 76 .

[14] D. Vizman, O. Grabner and G. Müller, J. Cryst. Growth 233 (2001) 687.

[15] D. Vizman, J. Friedrich and G. Müller, J. Cryst. Growth 303 (2007) 231.

[16] A. Popescu and D. Vizman, Cryst. Growth Des. 12 (2012) 320. 